\title{
Bifurcation and Basin in Two Coupled Parametrically Forced Logistic Maps
}

\author{
Hironori Kumeno \\ Yoshifumi Nishio \\ Daniele Fournier-Prunaret \\ Dept. of Electrical and Electronic Eng., Dept. of Electrical and Electronic Eng., LATTIS-INSA, Universite de Toulouse \\ Tokushima University, \\ Tokushima University, \\ 2-1 Minami-Josanjima, \\ 2-1 Minami-Josanjima, \\ Tokushima, 770-8506 JAPAN \\ Email: kumeno@ee.tokushima-u.ac.jp \\ Tokushima, 770-8506 JAPAN \\ 135 avenue de Rangueil, \\ 31077 Toulouse, France \\ Email: nishio@ee.tokushima-u.ac.jp \\ Email: daniele.fournier@insa-toulouse.fr
}

\begin{abstract}
Two coupled logistic maps whose parameters are forced into periodic varying are investigated. From the investigation of bifurcation in this system, nonexistence of odd periodic orbit except fixed point and existence of many coexisting attractors, which consist of periodic orbits or chaotic orbits, are observed. Basins where boundary depends on the invariant manifold of saddle points are numerically analyzed by considering second order iteration and using superposition with Newton method, although the system has discontinuity.
\end{abstract}

\section{INTRODUCTION}

There exists many incomprehensible relationship between brain mechanism and nonlinear phenomena of neuron and neural network. Although studies by using equipments which record global brain activity have possibilities, they are presently difficult. Therefore, we come up with the way which solves the problem by using model of brain with nonlinear systems. Neuron activity can be described by deterministic dynamical equation. So, it is possible to introduce models to investigate nonlinear neuronal activities and mutual influences between neurons in the neural networks by using nonlinear maps. When the real model which mimic brain activities can be analyzed, it does not only make progress on understanding of information processing mechanism of the brain, but also contributes to realize an information processing brain computer. So the brain activities can be realized on computers or special hardwares. It is known that if an autonomic neuron is influenced by external force, its characteristics are changed such as the neuron behaves periodic or chaotic [1]. How to give periodic external force is thought about in various ways, but we focus on a system whose parameter is forced into periodic varying and investigate behavior of the system and its pint-size coupling. The fundamental researches of these small coupled nonlinear systems are important for the investigation of global brain activities and the modeling.

As the research for a system with parameter forced into periodic varying, Ref. [2] reports that the almost periodic oscillation occurs in a simple oscillator including parametric excitation if nonlinear inductor has saturation characteristic. Additionally the occurrence of chaos is referred in Refs. [3] and [4]. Previously, we have investigated synchronization phenomena in three coupled logistic maps involving parametric force [5].

In this study, we investigate behavior of orbits which are periodic or chaotic in two coupled logistic maps whose parameters are forced into periodic varying. The paper is organized as follows. In the next section, we present the parametrically forced logistic map and coupling method used in this study. Bifurcation of the system is investigated in Section 3. In the investigation, nonexistence of odd periodic orbit except fixed point is confirmed. Section 4 illustrates basins when the parameter set corresponds to multistability phenomena. In the basins, fractal structures are confirmed at the corner of each basin. In section 5 , fixed point depending on the order of basin is numerically analyzed by using second order projection and its superposition. The last section is devoted to the conclusion.

\section{PARAMETRICALlY FORCED LOGISTIC MAP}

A parametrically forced logistic map used in this study is described as:

$$
x(n+1)=\alpha_{f}(n) x(n)(1-x(n)),
$$

and

$$
\alpha_{f}(n)= \begin{cases}\alpha_{1}, & \text { for each even value of " } n " \\ \alpha_{2}, & \text { for each odd value of " } n " \\ & (n=0,1,2, \ldots)\end{cases}
$$

where $\alpha_{f}(n)$ is a term of the parametric force and timevarying. In this system, two kinds of parameters, $\alpha_{1}$ and $\alpha_{2}$, are alternately replaced every update. Figure 1 shows an example of a return map of the parametrically forced logistic

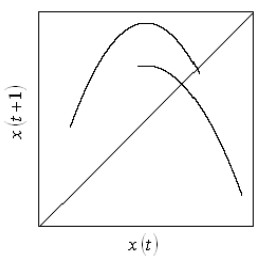

Fig. 1. Return map of parametrically forced logistic map for $\alpha_{1}=3.0$ and $\alpha_{2}=3.83$. 


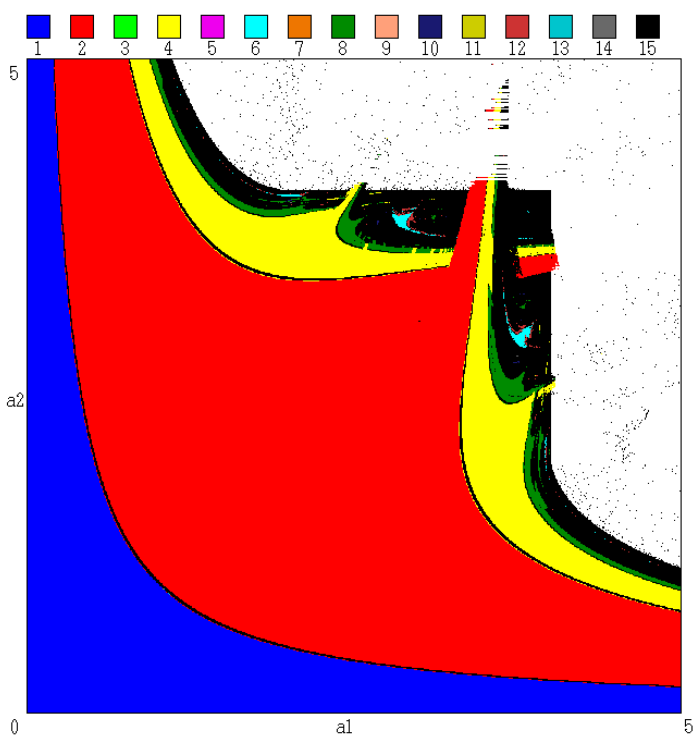

(a)

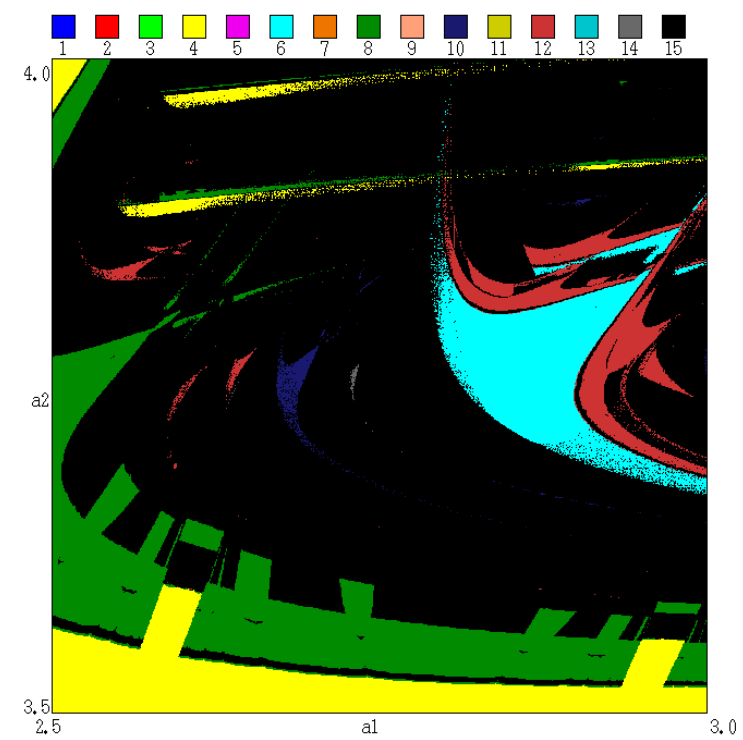

(b)

Fig. 2. Representation of the parameter plane $\left(\alpha_{1}, \alpha_{2}\right)$. Each colored part corresponds to the existence of a stable cycle (periodic point), the order $k$ (period) of which is given by the upper colored squares. The black color corresponds to $k \geq 15$ or to chaotic behavior. The white region corresponds to the nonexistence of attracting set, with chaotic transient toward infinity. Horizontal axis: $\alpha_{1}$. Vertical axis: $\alpha_{2}$. (b) Enlargement of (a)

map. For the original logistic map, two-periodic solution is observed for $\alpha=3.0$, while, three-periodic solution is observed for $\alpha=3.83$. These two solutions are periodic, whereas in the logistic map involving parametric force, a solution is chaotic as shown in Fig. 1 when the parameters $\alpha_{1}$ and $\alpha_{2}$ are set 3.0 and 3.83, respectively. Namely, chaotic solution can be observed in the combination of two parameters that generate two kinds of periodic solutions. This map is coupled with following coupling method:

$$
\begin{aligned}
& x_{i}(n+1)=(1-\varepsilon) f\left[x_{i}(n)\right]+\frac{\varepsilon}{N} \sum_{j=1}^{2} f\left[x_{j}(n)\right] \\
& i=1,2 .
\end{aligned}
$$

where $\varepsilon \in[0,1]$ is the coupling intensity. Then, the map $T$ is described as:

$$
T:\left\{\begin{aligned}
x_{1}(n+1)= & \left(1-\frac{\varepsilon}{2}\right) \alpha_{f} x_{1}(n)\left(1-x_{1}(n)\right) \\
& +\frac{\varepsilon}{2} \alpha_{f} x_{2}(n)\left(1-x_{2}(n)\right) \\
x_{2}(n+1)= & \left(1-\frac{\varepsilon}{2}\right) \alpha_{f} x_{2}(n)\left(1-x_{2}(n)\right) \\
& +\frac{\varepsilon}{2} \alpha_{f} x_{1}(n)\left(1-x_{1}(n)\right)
\end{aligned}\right.
$$

This study deals with the case that two parametrically forced logistic maps are coupled.

\section{BIFURCATION}

In this section, bifurcations are investigated with respect to $\alpha_{f}$. Figure 2 shows a two-parameter bifurcation diagram and its enlargement when $\varepsilon$ is fixed at 0.04 and $\alpha_{1}$ and $\alpha_{2}$ are changed. In the parameter $\left(\alpha_{1}, \alpha_{2}\right)$ plane, the existence domain of attracting set at finite distance is given by the (a)

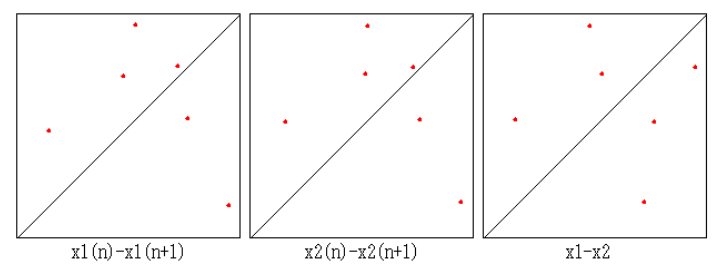

(b)

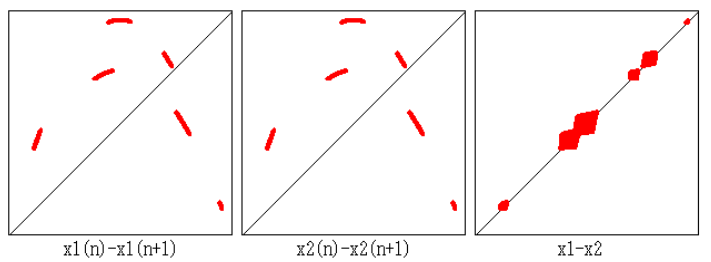

Fig. 3. return maps and phase differences in coexistence of period six orbit and chaotic orbit depending on initial values. $\alpha_{1}=2.94, \alpha_{2}=3.84$ and $\varepsilon=0.04$.

colored part of Fig. 2. Only even periodic orbits are observed except fixed point and the case of $\alpha_{1}=\alpha_{2}$. In the figure, some discontinuous boundaries of bifurcation are observed, for instance smooth boundary between green region and black region is intercepted by protuberant green region in Fig. 2(b). This discontinuity is caused due to multistability and initial conditions of calculation. In some regions, multistability behaviors can be observed. Different attractors can coexist, depending on the choice of different initial conditions. An example of the coexistence is shown in Fig. 3 when $\alpha_{1}=2.94$, $\alpha_{2}=3.84$ and $\varepsilon=0.04$. In this figure, period 6 orbit and chaotic orbit coexist, these orbits correspond to different initial values of $\left(x_{1}, x_{2}\right)$. 


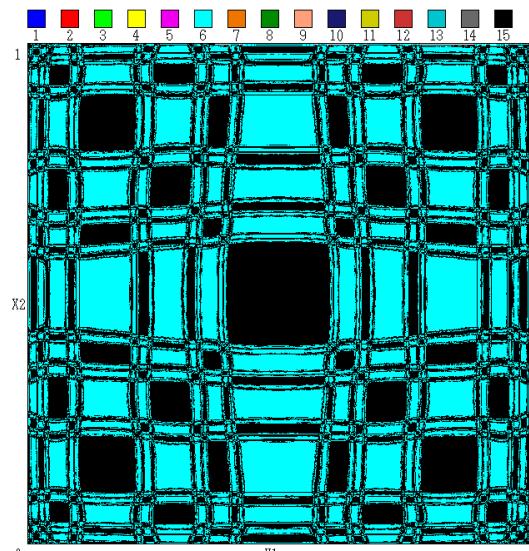

(a)

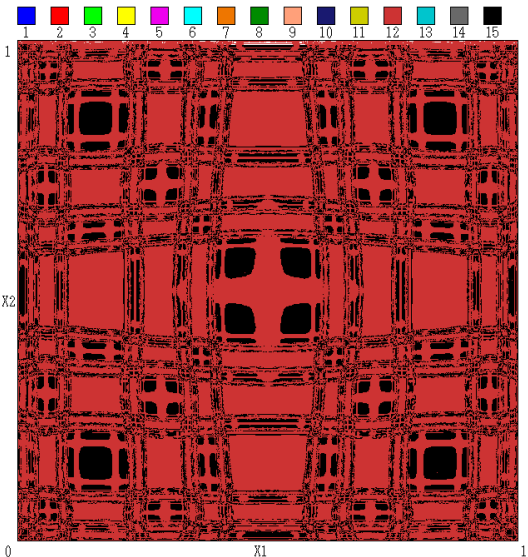

(b)

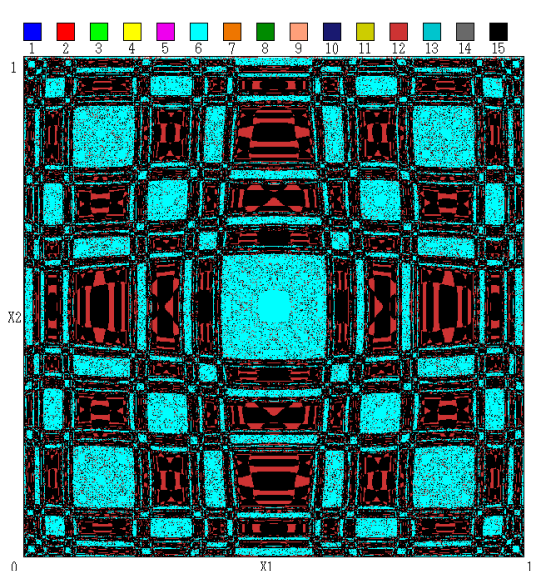

(c)

Fig. 4. Basin regarding to $T$ for $\alpha_{2}=3.84$ and $\varepsilon=0.04$. (a) $\alpha_{1}=2.94$. (b) $\alpha_{1}=2.97$. (c) $\alpha_{1}=2.98$. Horizontal axis: $x_{1}$. Vertical axis: $x_{2}$.

In the next section, basin which is an initial value set converging to a given orbit is investigated for parameter values corresponding to multistability.

\section{BASIN}

Depending on the property of the system, $\alpha_{f}$ is changed every update periodically. Figure 4 shows basin when the parameters $\alpha_{2}$ and $\varepsilon$ are considered as having fixed values, $\alpha_{1}$ varies with increasing values. Each basin of attraction, represented by a different color, corresponds to the period of orbit. Basins in Fig. 4(a) consist of the region having period 6 orbit, represented by cyan, and the region having chaotic orbit, represented by black. Increasing $\alpha_{1}$, basins are changing because there is coexistence of a period 12 orbit and a chaotic orbit. More increasing $\alpha_{1}$, we obtain three kinds of orbits, which are period 6 orbit, period 12 orbit and chaotic orbit.

In the basins, fractal structures are confirmed at the corner of each basin. Figures 5 show the enlargement around a corner of basin in Fig. 4(a) and its enlargements. With repeating enlargement, similar structure continues.

\section{PERIODIC ORBITS}

The parametrically forced logistic map is not continuous in iteration because $\alpha_{f}$ is periodically changed. Then, in order to obtain periodic orbits of $T$, that are all even, we can consider $T^{2}$. An order $k$ periodic orbit of $T^{2}$ will be an order $2 k$ periodic orbit of $T$. So we will obtain all even periodic orbits of $T$ by calculating all periodic orbits of $T^{2}$. To do this, we introduce $T_{\text {even }}$ and $T_{\text {odd }}$. We define the trajectory starting from the even number of $n$ as $T_{\text {even }}$, and the trajectory starting from the odd number of $n$ as $T_{o d d}$. All order $k$ periodic orbits of $T^{2}$ will be obtained by considering $T_{\text {even }}^{2}$ and $T_{\text {odd }}^{2}$ and by applying Newton method to the equations $T_{\text {even }}^{2}(X)=X$ and $T_{\text {odd }}^{2}(X)=X . T_{\text {even }}^{2}$ and $T_{\text {odd }}^{2}$ are continuous. Thus, Newton method can be applied. In Figure 6, dots indicate order 3 periodic orbits of $T^{2}$ which are detected by superimposing the order 3 periodic orbits calculated on $T_{\text {even }}^{2}$ and $T_{\text {odd }}^{2}$. Purple dots correspond to stable order 3 periodic orbit of $T^{2}$, red

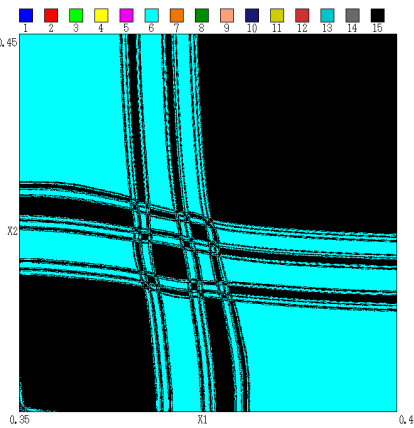

(a)

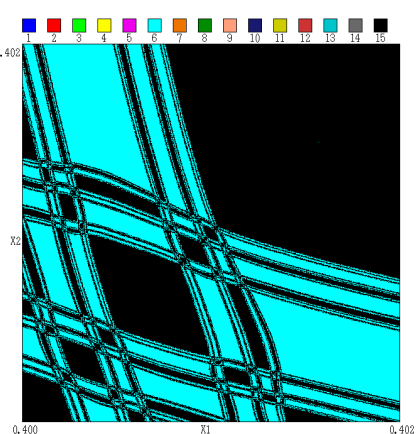

(c)

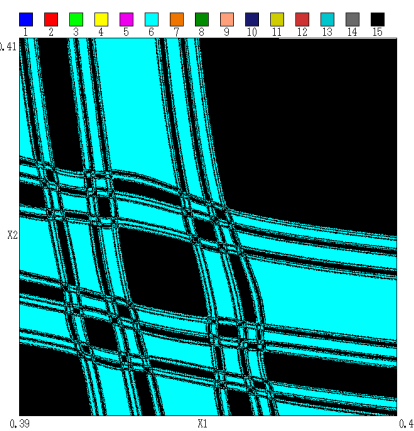

(b)

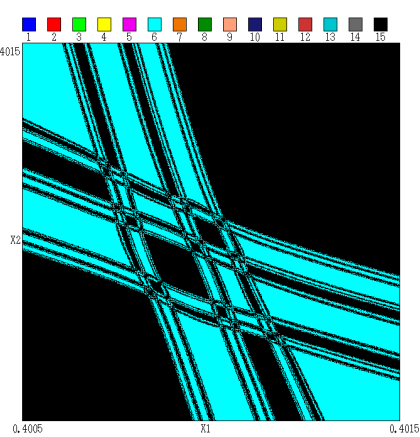

(d)
Fig. 5. Enlargement around a corner of basin. $\alpha_{1}=2.94, \alpha_{2}=3.84$ and $\varepsilon=0.04$. (a) Enlargement in [0.3, 0.4]. (b) Enlargement in [0.35, 0.38]. (c) Enlargement in [0.369, 0.365]. (d) Enlargement in [0.3615, 0.3625]. Fractal structures are shown.

points correspond to saddle, and yellow dots correspond to unstable node. Saddles are plotted on the corner of boundaries between basins. Then, it appears that stable manifold of saddle can be part of boundaries of basins.

\section{CONClusion}

In this study, we investigated a two-dimentional coupled logistic map whose parameters are forced into periodic varying. From the investigations of bifurcation, we confirmed that all 


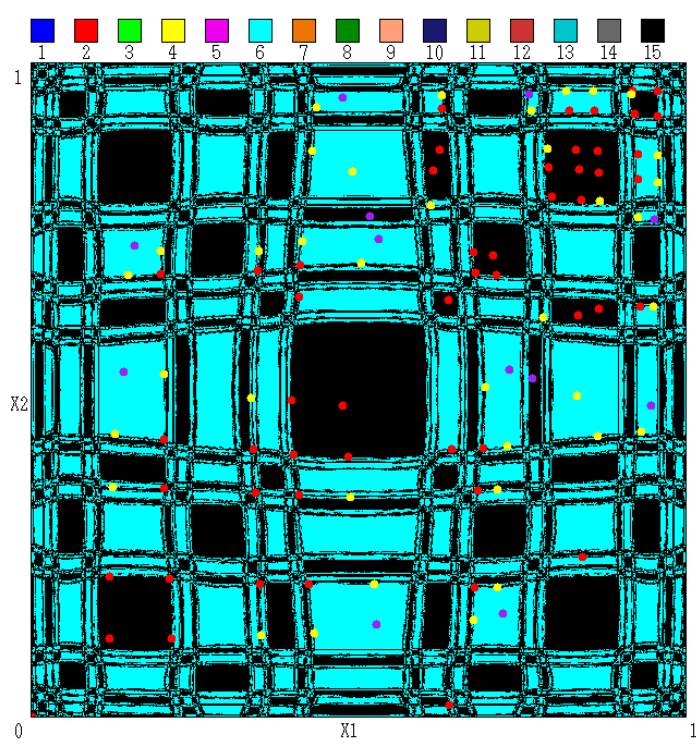

Fig. 6. Order 3 periodic orbits of $T^{2} . \alpha_{1}=2.94, \alpha_{2}=3.84$ and $\varepsilon=0.04$. Purple dots correspond to stable order 3 periodic orbit of $T^{2}$, red points correspond to saddle, and yellow dots correspond to unstable node.

the orbits correspond to only even order cycles except fixed point and the special case that $\alpha_{1}$ equals to $\alpha_{2}$. Moreover, existence of coexisting attractors, which consist of cycle orbit or chaotic orbit, is confirmed. Then, basin has been investigated when the coexistence occurs. In the basins, fractal structure appears. Periodic orbits and their basins are numerically calculated by using second order iteration and superposition with Newton method. By calculating its eigenvalues, it was confirmed that saddles are on the corner of boundaries between basins.

\section{REFERENCES}

[1] H. Hayashi, S. Ishizuka and K. Hirakawa "Transition to chaos via intermittency in the Onchidium pacemaker neuron." Physics letters. A, vol. 98, pp. 474-476, Nov, 1983.

[2] C. Hayashi, Nonlinear Oscillations in Physical Systems, Chap. 11, McGraw-Hill, New York 1964.

[3] C. Hayashi, M. Abe, K. Oshima and H. Kawakami, "The Method of Mapping as Applied to the Solution for Certain Types of Nonlinear Differential Equations," Proc. of the Ninth International Conference on Nonlinear Oscillations, pp. 1-8, 1981.

[4] M. Inoue, "A Method of Analysis for the Bifurcation of the Almost Periodic Oscillation and the Generation of Chaos in a Parametric Excitation Circuit," Trans. of IEICE, vol. J68-A, no. 7, pp. 621-626, 1985.

[5] H. Kumeno and Y. Nishio, "Globally Coupled Parametrically Forced Logistic Maps," Proc. of Asia Pacific Conference on Postgraduate Research in Microelectronics and Electronics (PrimeAsia'09), pp. 181-184, Nov. 2009. 